\title{
Racial and Ethnic Differences in Mortality Associated with Serum Potassium in Incident Peritoneal Dialysis Patients
}

\author{
Rieko Eriguchi ${ }^{a}$ Yoshitsugu Obji, ${ }^{a}$ Melissa Soohoo ${ }^{a}$ Connie M. Rhee \\ Csaba P. Kovesdy ${ }^{b, c}$ Kamyar Kalantar-Zadeh ${ }^{a, d, e}$ Elani Streja ${ }^{a, d}$ \\ ${ }^{a}$ Harold Simmons Center for Kidney Disease Research and Epidemiology, Division of Nephrology and Hypertension, \\ University of California Irvine, Orange, CA, USA; ${ }^{b}$ Division of Nephrology, University of Tennessee Health Science \\ Center, Memphis, TN, USA; ${ }^{C}$ Nephrology Section, Memphis Veterans Affairs Medical Center, Memphis, TN, USA;

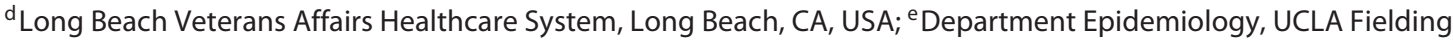 \\ School of Public Health, University of California Los Angeles, Los Angeles, CA, USA
}

\section{Keywords}

Serum potassium · Peritoneal dialysis · Mortality $\cdot$ Race/ ethnicity

\begin{abstract}
Background: Abnormalities in serum potassium are risk factors for sudden cardiac death and arrhythmias among dialysis patients. Although a previous study in hemodialysis patients has shown that race/ethnicity may impact the relationship between serum potassium and mortality, the relationship remains unclear among peritoneal dialysis (PD) patients where the dynamics of serum potassium is more stable. Methods: Among 17,664 patients who started PD between January 1, 2007 and December 31, 2011 in a large US dialysis organization, we evaluated the association of serum potassium levels with all-cause and arrhythmia-related deaths across race/ethnicity using time-dependent Cox models with adjustments for demographics. We also used restricted cubic spline functions for serum potassium levels to explore non-linear associations. Results: Baseline serum potassium levels were the highest among Hispanics (4.2 \pm $0.7 \mathrm{mEq} / \mathrm{L})$ and lowest among non-Hispanic blacks (4.0 \pm 0.7 $\mathrm{mEq} / \mathrm{L}$ ). Among 2,949 deaths during the follow-up of median
\end{abstract}

\section{KARGER}

(c) 2019 S. Karger AG, Basel

E-Mail karger@karger.com

www.karger.com/ajn
2.2 (interquartile ranges 1.3-3.2) years, $683(23 \%)$ were arrhythmia-related deaths. Overall, both hyperkalemia and hypokalemia (i.e., serum potassium levels $>5.0$ and $<3.5 \mathrm{mEq} / \mathrm{L}$, respectively) were associated with higher all-cause and arrhythmia-related mortality. In a stratified analysis according to race/ethnicity, the association of hypokalemia with allcause and arrhythmia-related mortality was consistent with an attenuation for arrhythmia-related mortality in non-Hispanic blacks. Hyperkalemia was associated with all-cause and arrhythmia-related mortality in non-Hispanic whites and non-Hispanic blacks, but no association was observed in Hispanics. Conclusion: Among incident PD patients, hypokalemia was consistently associated with all-cause and arrhythmia-related deaths irrespective of race/ethnicity. However, while hyperkalemia was associated with both death outcomes in non-Hispanic blacks and whites, it was not associated with either death outcome in Hispanic patients. Further studies are needed to demonstrate whether different strategies should be followed for the management of serum potassium levels according to race/ethnicity.

(C) 2019 S. Karger AG, Basel

R.E. and Y.O. equally contributed to the manuscript. 


\section{Introduction}

Cardiovascular disease is the leading cause of death among dialysis patients [1,2], where arrhythmia and cardiac arrest are some of the major causes of cardiovascular deaths. Abnormalities in serum potassium levels (i.e., hyperkalemia and hypokalemia; or dyskalemias) are risk factors for arrhythmias and sudden cardiac death [3, 4]. Hypokalemia may lead to ventricular fibrillation $[5,6]$, while hyperkalemia can lead to electromechanical dissociation and asystole $[7,8]$. Dyskalemias are common among dialysis patients $[9$, 10], and both high and low serum potassium levels are associated with higher mortality risk [11]. Although peritoneal dialysis (PD), when compared to hemodialysis, is less likely to induce myocardial stunning due to slower rates of solute clearance and fluid removal [12], the prevalence of cardiac arrest and ventricular arrhythmias is actually similar between these 2 dialysis modalities [1]. This contradictory consequence may be due to lower serum potassium levels among PD patients [11].

Several studies have examined the impact of race/ethnicity on the association of serum potassium levels with mortality; however, the findings were inconsistent. One study of non-dialysis dependent chronic kidney disease (CKD) patients found that hyperkalemia was associated with a higher death risk among whites but not among African Americans, while hypokalemia was consistently associated with mortality in both races [13]. Similarly, a later study with over 2 million non-CKD US veterans found that mortality risk associated with hyperkalemia was lower among African Americans than among nonAfrican Americans [14]. In maintenance hemodialysis patients, however, higher baseline potassium levels were associated with higher mortality in both non-Hispanic whites and non-Hispanic blacks but not in Hispanics [15]. Despite these prior studies, it remains unclear whether race/ethnicity modifies the risk associated with serum potassium concentrations for all-cause death or arrhythmia-related death among PD patients, considering that potassium kinetics are different among hemodialysis and PD patients. For example, serum potassium abruptly decreases during hemodialysis treatment and gradually increases thereafter, whereas concentrations are more stable among PD patients. We therefore hypothesized that the impact of serum potassium levels on mortality is also different according to race/ethnicity among PD patients.

\section{Materials and Methods}

The study was approved by the Institutional Review Committees of the University of California, Irvine and Los Angeles Biomedical Research Institute at Harbor-UCLA. Given the large sample size, anonymity of the patients studied, and nonintrusive nature of the research, the requirement for written consent was exempt.

\section{Patients}

We retrospectively analyzed a statistically de-identified data set from a large dialysis organization in the United States [16]. We analyzed 19,844 patients aged 18 years or older who initiated and received $\mathrm{PD}$ from one of the facilities operated by the dialysis provider over a 5-year period (January 1, 2007 to December 31, 2011). We excluded 713 patients without available serum potassium data while they were treated with $\mathrm{PD}$, and then 1,467 patients of other race/ethnicities (767 Asian patients and 700 patients of other races) than that of non-Hispanic whites, non-Hispanic blacks, and Hispanics, resulting in the final analytical cohort of 17,664 incident PD patients (online suppl. Appendix Fig. S1; for all online suppl. material, see www.karger.com/doi/10.1159/000502998). Patients were followed from their PD start until the occurrence of one of the following events: primary outcome (death), kidney transplantation, transfer to another dialysis modality or to a facility operated by another dialysis provider, or end of administrative follow-up (December 31, 2011).

\section{Demographic, Clinical, and Laboratory Measures}

Information on death events, causes of death, race/ethnicity, primary insurance, access type, presence of comorbidities, and laboratory variables were obtained from the administrative electronic database of the dialysis provider. The specific causes of death code (i.e., hypokalemia, hyperkalemia, cardiac arrhythmia, and cardiac arrest) were sourced from data from the large dialysis organization. Blood samples were drawn in dialysis facilities and shipped to a central laboratory, typically within $24 \mathrm{~h}$. All laboratory values were measured by automated and standardized methods. To minimize measurement variability, all repeated measures of laboratory variables for each patient were averaged monthly from time of PD start. The averaged values during the first month after PD start served as baseline data.

\section{Statistical Analyses}

We examined baseline clinical characteristics across race/ethnicity as well as according to baseline low $(<3.5 \mathrm{mEq} / \mathrm{L}$ : hypokalemia), medium (3.5-5.0 mEq/L: normal), and high (>5.0 mEq/L: hyperkalemia) serum potassium levels within each race/ethnicity group. Data are presented as means $( \pm S D)$ or medians (interquartile ranges), or proportions, where appropriate.

The exposure of interest was monthly averaged serum potassium levels. Between 70 and $80 \%$ of monthly averaged potassium values were derived from only one measurement (online suppl. Appendix Fig. S2). The primary outcome of interest was all-cause mortality. The secondary outcome was the composite outcome of arrhythmia-related deaths or those potentially related to dyskalemias (i.e., deaths due to hypokalemia, hyperkalemia, cardiac arrhythmia, and cardiac arrest). As a sensitivity analysis, we also examined associations with any cardiac death (i.e., myocardial infarction, pericarditis, atheroscleroticheartdisease, cardiomyopathy, cardiac arrhythmia, cardiac arrest, valvular heart disease, pulmo- 
nary edema, and congestive heart disease). Moreover, we also examined the association of the highest and lowest measured potassium levels of each month with mortality because the use of monthly averaged values may bias estimates toward the null if serial serum potassium levels were measured after the intervention against dyskalemias.

We used time-dependent Cox models to examine the shortterm association between serum potassium levels and mortality. Monthly averaged serum potassium levels were modeled as continuous variables, and the non-linear relationship with each outcome was estimated using restricted cubic spline functions with 4 knots at the 5th, 35th, 65th, and 95th percentiles of observed values and using serum potassium $4.0 \mathrm{mEq} / \mathrm{L}$ as a reference. We also used post estimation to calculate predicted hazard ratios at serum potassium levels of 2.5, 3.0, 3.5, 4.5, 5.0, 5.5, 6.0, 6.5, and $7.0 \mathrm{mEq} / \mathrm{L}$ using $4.0 \mathrm{mEq} / \mathrm{L}$ as a reference. We employed 3 levels of hierarchical adjustment: (1) unadjusted model, (2) case-mix adjusted model that included age, sex, race/ethnicity, diabetes mellitus, and primary insurance at baseline as well as the preexisting comorbidities (hypertension, atherosclerotic heart disease, congestive heart failure, and other cardiovascular disease), and (3) case-mix and malnutrition-inflammation cachexia syndrome (MICS) adjusted model that included all above-mentioned covariates and time-updated data on 7 surrogates of nutritional and inflammatory status; body mass index, hemoglobin, and serum levels of albumin, creatinine, calcium, phosphorus, and bicarbonate. We used log-likelihood ratio tests to formally assess the interaction between time varying serum potassium and race ethnicity with mortality.

For evaluating differences in hazard ratios across race/ethnicity, we evaluated the association under the unadjusted and casemix adjusted models as our primary model due to potential over adjustment with MICS variables because of the limited number of events. However, in sensitivity analysis, we also evaluated associations with the case-mix model and additional adjustment for serum creatinine, $\mathrm{Kt} / \mathrm{V}$, and residual renal function (RRF). Missing covariate data at baseline and during the follow-up period were imputed using the mean of within-individual values measured within \pm 1 month, or using the last-observation carried forward method if no data were available during \pm 1 month. Missing frequencies at baseline (i.e., the first month) and during the first 2 months (i.e., the first and second month) were $<10$ and $<1 \%$ for hemoglobin, serum albumin, calcium, and phosphorus; 15 and $1.5 \%$ for serum creatinine and bicarbonate; and 50 and 19\% for body mass index, respectively. We also conducted sensitivity analyses after restricting the study population to 16,004 patients with available data on baseline (i.e., the first month from PD start) serum potassium. All analyses were carried out using STATA MP version 13.1 (StataCorp., College Station, TX, USA).

\section{Results}

Baseline Demographic, Clinical, and Laboratory

Characteristics According to Baseline Serum

Potassium Level Across Race/Ethnicity

Among 17,664 patients, the mean \pm SD age was $56 \pm$ 16 years; $44 \%$ were female; $62 \%$ were non-Hispanic white, $25 \%$ were non-Hispanic black, and $14 \%$ were Hispanic; and $62 \%$ had diabetes as the cause of end-stage renal disease (Table 1). Baseline serum potassium levels were the highest among Hispanics $(4.2 \pm 0.7 \mathrm{mEq} / \mathrm{L})$ and lowest among non-Hispanic blacks ( $4.0 \pm 0.7 \mathrm{mEq} / \mathrm{L} ; p<0.001)$. The prevalence of hyper- and hypokalemia (i.e., serum potassium levels $>5.0$ and $<3.5 \mathrm{mEq} / \mathrm{L}$ ) was 9 and $15 \%$ among non-Hispanic whites, 8 and $20 \%$ among non-Hispanic blacks, and 12 and 13\% among Hispanics respectively (online suppl. Appendix Table 1). Those who had lower serum potassium levels in all races were more likely to be older, female; have higher bicarbonate; and have lower serum albumin, creatinine, and phosphorus.

\section{Association of Monthly-Averaged Serum}

Potassium Levels with All-Cause Mortality and

Arrhythmia-Related Mortality in the Entire Cohort

A total of 2,949 deaths, including 683 arrhythmia-related deaths ( $90 \%$ of which were coded as cardiac arrest), were observed during the follow-up period (up to 5 years; median 2.2 [interquartile ranges 1.3-3.2] years). In spline models, lower serum potassium was associated with higher risk of all-cause and arrhythmia-related death across all levels of adjustment, while associations of higher serum potassium with higher risk of both death outcomes attenuated towards the null with model adjustment (Fig. 1).

In the unadjusted model, both hyperkalemia and hypokalemia were associated with higher all-cause mortality compared with the referent value $(4.0 \mathrm{mEq} / \mathrm{L}$; Fig. 1a). These findings were consistent in the primary model (i.e., the case-mix adjusted model) with hazard ratios (HRs; 95\% CI) of 4.16 (3.40-5.08), 1.41 (1.35-1.47), 1.15 (1.06$1.25)$, and $1.65(1.41-1.93)$ at serum potassium levels of $2.5,3.5,5.0$, and $6.0 \mathrm{mEq} / \mathrm{L}$ (vs. $4.0 \mathrm{mEq} / \mathrm{L}$ ) respectively (Fig. 1b). Further adjustment for nutritional and inflammatory surrogates (i.e., the case-mix and MICS model) attenuated these associations, but the association of hypokalemia with all-cause mortality remained similar; HRs (95\% CI) were 2.20 (1.66-2.93), 1.64 (1.39-1.94), and $1.22(1.15-1.30)$ at serum potassium level of 2.5, 3.0, and $3.5 \mathrm{mEq} / \mathrm{L}$ respectively (Fig. 1c). Associations of hyperkalemia with all-cause mortality were attenuated in the case-mix and MICS model.

Serum potassium levels showed a similar pattern in its associations with arrhythmia-related mortality (vs. allcause mortality; Fig. 1d-f). Case-mix adjusted HRs (95\% CI) were 4.43 (2.95-6.66), 1.39 (1.28-1.52), 1.23 (1.03$1.46)$, and $1.64(1.18-2.27)$ at serum potassium level of 2.5, 3.5, 5.0 and $6.0 \mathrm{mEq} / \mathrm{L}$ respectively (Fig. 1e). Further adjustment for nutritional and inflammatory surrogates (i.e., the case-mix and MICS model) attenuated these as- 
Table 1. Baseline characteristics stratified race/ethnicity in 17,664 incident PD patients

\begin{tabular}{|c|c|c|c|c|}
\hline Variable & Overall & non-hispanic white & non-hispanic black & hispanic \\
\hline Age, years & $56 \pm 16$ & $60 \pm 15$ & $51 \pm 14$ & $51 \pm 16$ \\
\hline Gender, female, \% & 44 & 40 & 52 & 44 \\
\hline $\mathrm{DM}, \%$ & 62 & 60 & 62 & 70 \\
\hline Medicaid & 4 & 3 & 4 & 11 \\
\hline Others & 49 & 48 & 54 & 45 \\
\hline \multicolumn{5}{|l|}{ Comorbidities, \% } \\
\hline Hypertension & 53 & 50 & 63 & 47 \\
\hline $\mathrm{CHF}$ & 20 & 19 & 22 & 18 \\
\hline ASHD & 17 & 19 & 14 & 13 \\
\hline Potassium, mEq/L & $4.1 \pm 0.7$ & $4.1 \pm 0.7$ & $4.0 \pm 0.7$ & $4.2 \pm 0.7$ \\
\hline $\mathrm{WBC}, 1,000 / \mu \mathrm{L}$ & $7.0(5.7-8.7)$ & $7.3(5.9-8.9)$ & $6.4(5.1-7.9)$ & $7.1(5.9-8.9)$ \\
\hline Hemoglobin, g/dL & $11.6 \pm 1.5$ & $11.7 \pm 1.5$ & $11.4 \pm 1.6$ & $11.7 \pm 1.6$ \\
\hline Albumin, g/dL & $3.7 \pm 0.5$ & $3.7 \pm 0.5$ & $3.7 \pm 0.5$ & $3.7 \pm 0.6$ \\
\hline Creatinine, $\mathrm{mg} / \mathrm{dL}$ & $7.1 \pm 3.6$ & $6.3 \pm 3.1$ & $8.6 \pm 4.3$ & $7.6 \pm 3.8$ \\
\hline Calcium, mg/dL & $8.8 \pm 0.8$ & $8.9 \pm 0.7$ & $8.8 \pm 0.8$ & $8.6 \pm 0.8$ \\
\hline Phosphorus, mg/dL & $5.0 \pm 1.5$ & $5.0 \pm 1.5$ & $5.0 \pm 1.5$ & $5.1 \pm 1.5$ \\
\hline Bicarbonate, mEq/L & $25.2 \pm 3.5$ & $25.5 \pm 3.5$ & $25.0 \pm 3.5$ & $24.7 \pm 3.5$ \\
\hline
\end{tabular}

Continuous values are expressed as mean \pm SD or median (interquartile range) as appropriate.

Missing frequency at consecutive months (month 1 and 2 ) was $1.5 \%$ for serum creatinine, $8.1 \%$ for BMI, and $<1 \%$ for hemoglobin, serum albumin, calcium, phosphorus, and bicarbonate.

$\mathrm{PD}$, peritoneal dialysis; DM, diabetes mellitus; $\mathrm{CHF}$, congestive heart failure; ASHD, atherosclerotic heart disease; BMI, body mass index; WBC, white blood cell.

sociations, but the association of hypokalemia with arrhythmia-related mortality remained significant; HRs (95\% CI) were 2.36 (1.31-4.24), 1.70 (1.21-2.40), and 1.24 $(1.09-1.40)$ at serum potassium level of 2.5, 3.0, and $3.5 \mathrm{mEq} / \mathrm{L}$ respectively (Fig. 1f). Associations of hyperkalemia with arrhythmia-related mortality were attenuated in the case-mix and MICS model.

\section{Association of Monthly-Averaged Serum Potassium}

Levels with All-Cause Mortality Across Race/Ethnicity

During the follow-up period, 2,216, 488, and 245 allcause deaths were observed among non-Hispanic white, non-Hispanic black, and Hispanic patients, respectively. Among non-Hispanic white patients, serum potassium levels showed a U-shaped association with higher allcause mortality (case-mix adjusted HRs, 4.98 [3.88-6.40], 1.47 [1.40-1.55], 1.17 [1.06-1.30], and 1.79 [1.49-2.14] at $2.5,3.5,5.0$, and $6.0 \mathrm{mEq} / \mathrm{L}$ [referent, $4.0 \mathrm{mEq} / \mathrm{L}$ ], respec- tively; Fig. 2a). A slightly attenuated but consistent association was observed among non-Hispanic black patients with case-mix adjusted HRs of 2.50 (1.57-3.97), 1.19 (1.07-1.31), $1.27(1.02-1.57)$, and $1.37(1.01-1.85)$ at 2.5 , 3.5, 5.0, and $5.5 \mathrm{mEq} / \mathrm{L}$, respectively (Fig. $2 \mathrm{~b}$ ). Among Hispanic patients, lower serum potassium levels were consistently associated with higher all-cause mortality, but the mortality risk was not elevated at higher serum potassium levels (case-mix adjusted HRs, 5.37 [2.979.73], 2.84 [2.01-4.00], and 1.51 [1.32-1.73] at 2.5, 3.0, and $3.5 \mathrm{mEq} / \mathrm{L}$, respectively; Fig. 2c). Under the case-mix, serum creatinine, Kt/V, and RRF adjusted model, the association estimates were modestly attenuated, but showed a similar pattern to the main results (online suppl. Appendix Fig. S3). Log likelihood tests demonstrated significant interactions between race-ethnicity and serum potassium for mortality outcomes (case-mix adjusted model $[p<0.001]$, case-mix and MICS model $[p=0.035]$ ). 

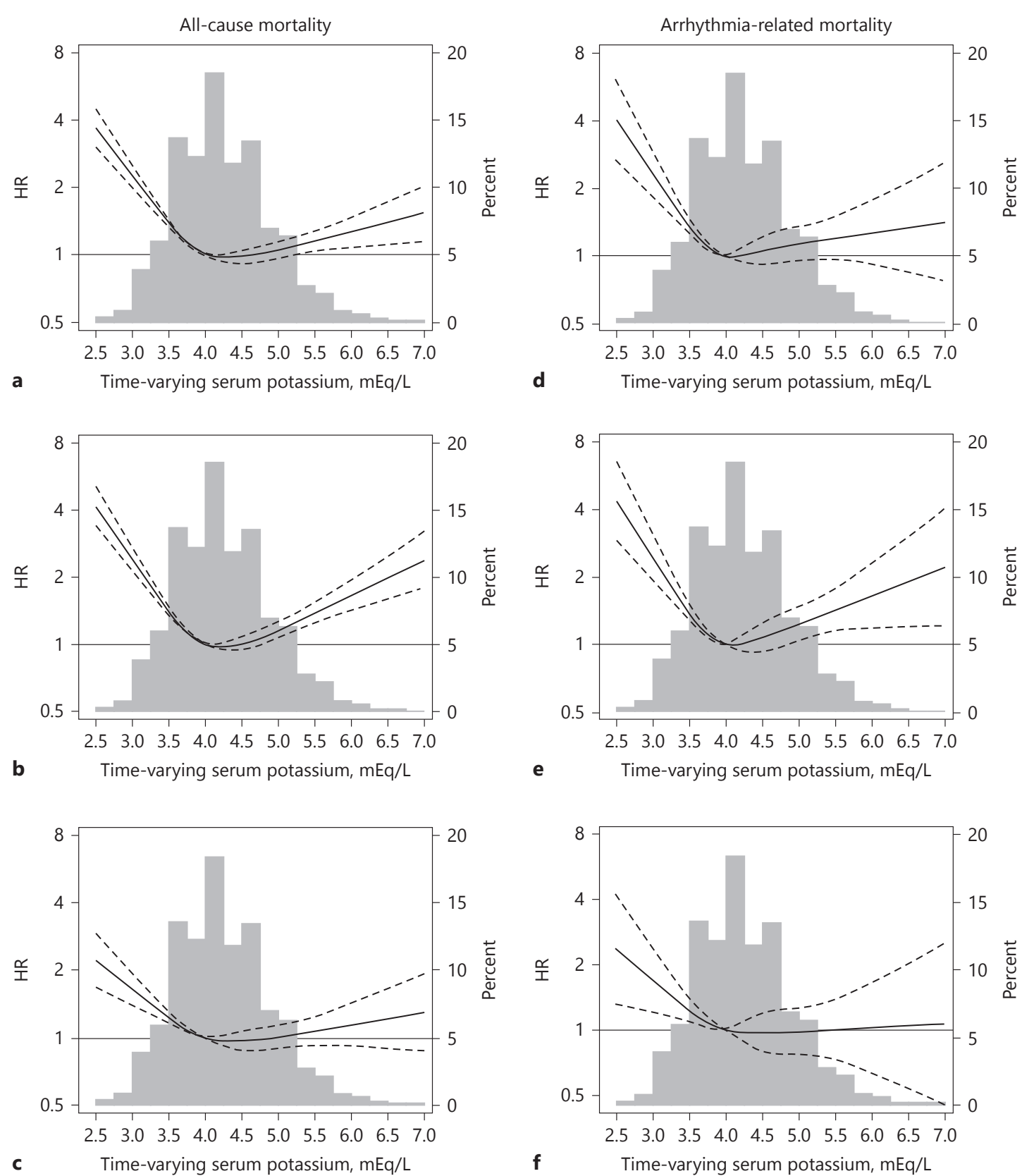

Fig. 1. Distribution of baseline serum potassium levels and the association of monthly averaged serum potassium levels with (a-c) all-cause mortality and (d-f) arrhythmia-related mortality with 3

levels of adjustment (unadjusted, case-mix adjusted, and case-mix and MICS adjusted). Dashed and solid lines represent HRs estimates and 95\% CIs, respectively. HR, hazard ratios.

\section{Association of Monthly-Averaged Serum Potassium \\ Levels with Arrhythmia-Related Mortality Across \\ Race/Ethnicity}

During the follow-up period, 486, 116, and 81 arrhythmia-related deaths were observed among non-Hispanic white, non-Hispanic black, and Hispanic patients respec-

tively. Consistent findings were obtained for arrhythmiarelated death as compared to all-cause death (Fig. 3). Higher serum potassium levels were associated with higher arrhythmia-related mortality among non-Hispanic white and non-Hispanic black patients but not among Hispanic patients. The association between hypokalemia 


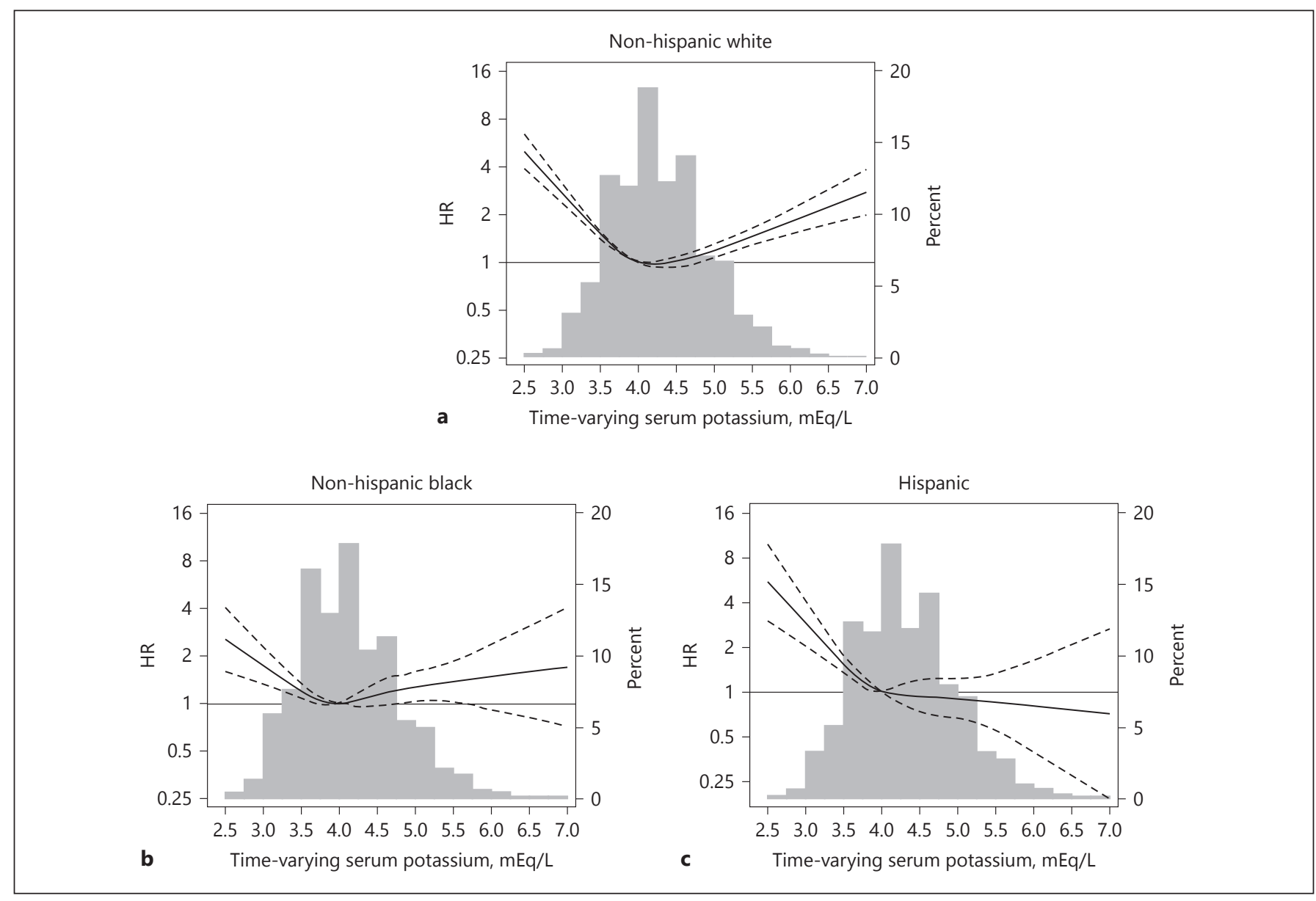

Fig. 2. Distribution of baseline serum potassium levels and the association of monthly averaged serum potassium levels with allcause mortality across race/ethnicity in the case-mix adjusted

$<3.5 \mathrm{mEq} / \mathrm{L}$ and arrhythmia-related mortality appeared consistent across race/ethnicity although it was attenuated for non-Hispanic black patients, possibly due to a limited number of events. The association of serum potassium levels with arrhythmia-related mortality across race/ethnicity was modestly attenuated under case-mix, serum creatinine, Kt/V, and RRF adjustments, however, the relationships were consistent (online suppl. Appendix Fig. S4). We also observed a consistent race/ethnic difference in the association of serum potassium levels with cardiac death (online suppl. Appendix Fig. S5).

Using the highest and lowest serum potassium levels as exposures, we also observed consistent results across race/ethnicity. Sensitivity analyses after restricting the study population to 16,004 patients with available serum potassium data at baseline (i.e., the first month from PD start) also showed consistent results. model; (a) non-Hispanic white, (b) non-Hispanic black, and (c) Hispanic patients. Dashed and solid lines represent HRs estimates and $95 \%$ CIs respectively. HR, hazard ratios.

\section{Discussion}

In this longitudinal national cohort of 17,664 incident PD patients, we demonstrated that lower serum potassium levels $<3.5 \mathrm{mEq} / \mathrm{L}$ were consistently associated with higher all-cause and arrhythmia-related deaths across race/ethnicity. On the other hand, higher serum potassium levels $>5.0 \mathrm{mEq} / \mathrm{L}$ were associated with all-cause and arrhythmia-related death among non-Hispanic whites and blacks but not among Hispanic patients. To our knowledge, this is the first study to examine the differences in the impact of serum potassium levels on mortality across racial/ethnic groups among PD patients using a time-dependent model.

Hypokalemia was a risk factor for all-cause death among PD patients in our study. Short-term associations with mortality, as indicated by the time-dependent model, could 


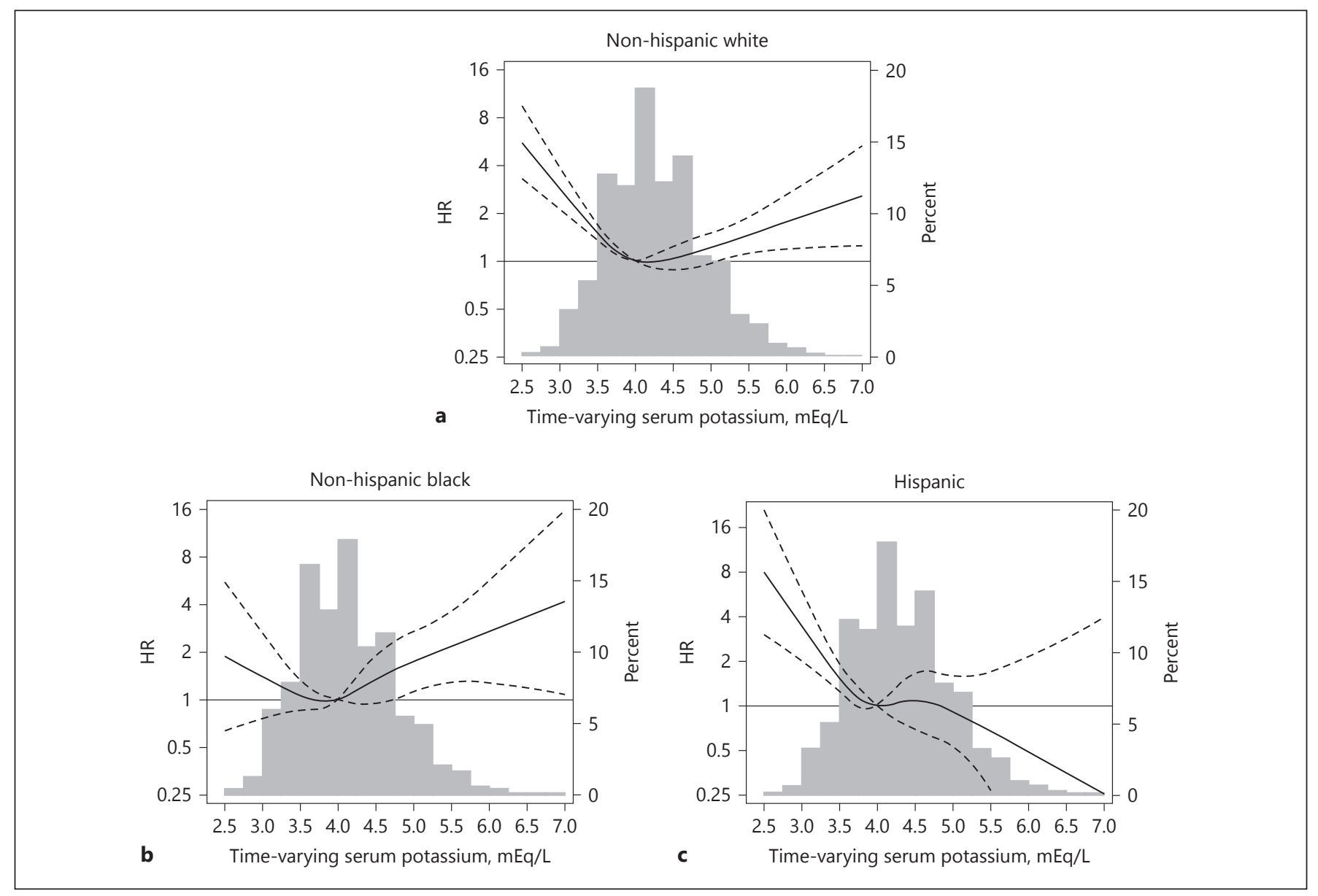

Fig. 3. Distribution of baseline serum potassium levels and the association of monthly averaged serum potassium levels with arrhythmia-related mortality across race/ethnicity in the case-mix adjusted model; (a) non-Hispanic white, (b) non-Hispanic black, and (c) Hispanic patients. Dashed and solid lines represent HRs estimates and 95\% CIs respectively. HR, hazard ratios. be partly explained by the impact of potassium on ventricular arrhythmogenicity $[17,18]$. Indeed, we confirmed the association with arrhythmia-related death even after adjusting for various nutritional and inflammatory markers. Other potential factors contributing to a hypokalemiamortality association include predisposition to stroke [19] and respiratory muscle weakness [20], but there still may be residual confounding with MICS [11], given the correlation between oral intakes of potassium and protein.

In this study, serum potassium levels were the lowest among non-Hispanic blacks and the highest among Hispanics, with non-Hispanic whites in the middle. This tendency was also observed in previous studies among healthy adults [14] and hemodialysis patients [15]. It has been shown that dietary intake and urinary excretion of potassium are different according to race/ethnicity [21, 22]. For example, non-Hispanic black patients consume a lower potassium diet [23] and have significantly lower urinary potassium excretion [24-27]. Although serum potassium level has a poor correlation with dietary potassium intake in the dialysis population $[28,29]$, residual kidney function among PD patients may still play a role in the difference in serum potassium levels; the lower urinary excretion of potassium in blacks seemed to be independent of dietary differences in the Dietary Approaches to Stop Hypertension trial [27]. Other possible explanations for the differences in potassium dynamics among non-Hispanic blacks include (1) increased activity of the $\mathrm{Na}-\mathrm{K}-2 \mathrm{Cl}$ cotransporter in the thick ascending limb of the loop of Henle [24], (2) increased fecal excretion of potassium [30], and (3) differences in cellular potassium disposal [25]. Gastrointestinal absorption [31] or aldosterone excretion [32] may also be related to racial differences in serum potassium levels. 
We found a racial/ethnic difference in the impact of hyperkalemia on all-cause and arrhythmia-related mortality among PD patients. One possible explanation is the difference in the trajectories of serum potassium levels before developing hyperkalemia across race/ethnicity. Chronic hyperkalemia is reported to be better tolerated than an acute rise, and patients with CKD who chronically increase their serum potassium levels can present with no apparent electrocardiographic or cardiovascular manifestations even in the range of $>6.0 \mathrm{mEq} / \mathrm{L}$ [33-35]. The highest baseline serum potassium levels among Hispanic PD patients indicates the more chronic nature of hyperkalemia after dialysis initiation than the other groups, which might have led to tolerance against hyperkalemia. Another potential mechanism for the racial/ethnic difference in the hyperkalemia-mortality association is the racial/ethnic difference in cardiac potassium channel variants that may be related to susceptibility against dyskalemia [36]. In contrast to the association between hyperkalemia and mortality, however, our study in PD patients suggested that lower potassium levels were consistently associated with all-cause and arrhythmia-related death across race/ethnicity. Some recent studies showed that a greater fruit and vegetable intake was associated with improving cardiovascular disease risk indicators among patients with CKD [37], and lower allcause and non-cardiovascular death among maintenance hemodialysis patients [38]. Hispanics may be good candidates for liberalizing potassium intake, which requires future investigation.

Several limitations of our study should be noted. First, given the nature of an observational cohort analysis, there may be residual confounding or unmeasured confounders. Second, the small numbers of outcome events might have limited our ability to detect the arrhythmia-mortality association. However, it should be noted that the estimated HRs for both all-cause and arrhythmia-related death at higher serum potassium levels were lower than 1.0 among Hispanic patients. Third, information on the cause of death was recorded by the LDO and we cannot ascertain its accuracy and precision. The majority of arrhythmia-related deaths were due cardiac arrest, which does not differentiate between bradycardia and tachycardia. Cardiac arrest in arrhythmia-related or cardiac death may include the other situations that are not related with abnormal serum potassium levels while arrhythmia-related or cardiac deaths might have been categorized as other causes of death. Additionally, very few patients had hypokalemia and hyperkalemia listed as the specific cause of death and we therefore could not separately evaluate associations with these more specific directional causes of death. Further studies are needed to examine these relationships. Fourth, we did not have precise data on medications which could have influenced serum potassium levels and all-cause or arrhythmia-related death.

In conclusion, our study demonstrated that among incident PD patients, hypokalemia was consistently associated with all-cause and arrhythmia-related deaths irrespective of race/ethnicity while hyperkalemia was not associated with either mortality outcome in Hispanics. Further studies are needed to demonstrate whether different strategies should be followed in the management of serum potassium levels according to race/ethnicity.

\section{Acknowledgments}

DaVita Clinical Research provided a statistically de-identified data set for this study. The interpretation and conclusions are those of the authors and do not represent the views of DaVita, Inc. The results presented in this paper have not been published previously in whole or part, except in abstract format. The study was supported by National Institute of Diabetes, Digestive and Kidney Disease (NIDDK) of the National Institute of Health (NIH; K24-DK091419 to K.K.-Z.) and philanthropic grants from Mr. Harold Simmons, Mr. Louis Chang, Dr. Joseph Lee and AVEO, Inc. (to K.K.-Z.). E.S.: is supported by Office of Research and Development of the Department of Veterans Affairs career development award IK2-CX001266-01. C.M.R.: is supported by NIH (NIDDK) grant K23-DK102903, and C.P.K.: is supported by NIH (NIDDK) U01-DK102163.

\section{Disclosure Statement}

C.P.K. has received honoraria from Astra Zeneca (Wilmington, DE, USA), Bayer (Whippany, NJ, USA), Dr. Schar and SanofiAventis (Bridgewater, NJ, USA), and grant support from Shire (Lexington, MA, USA). K.K.-Z. has received commercial honoraria and/or support from Abbott (Abbott Park, IL, USA), Abbvie (North Chicago, IL, USA), Alexion (New Haven, CT, USA), Amgen (Thousand Oaks, CA), Astra-Zeneca (Wilmington, DE, USA), Aveo (Cambridge, MA, USA), Chugai (Berkeley Heights, NJ, USA), Fresenius (Waltham, MA, USA), Genentech (South San Francisco, CA, USA), Haymarket Media (London, England), Hospira (Lake Forest, IL, USA), Kabi (Lake Zurich, IL, USA), Keryx (Boston, MA, USA), Novartis (New York, NY, USA), Pfizer (New York, NY, USA), Relypsa, Resverlogix (Calgary, Alberta, Canada), Sandoz (Princeton, NJ, USA), Sanofi-Aventis (Bridgewater, NJ, USA), Shire (Lexington, MA, USA), Vifor (Zurich, Switzerland), UpToDate (Waltham, MA, USA), and ZS Pharma (Coppell, TX, USA).

\section{Funding Sources}

The study was supported by NIDDK of the NIH (K24-DK091419 to K.K.-Z.) and philanthropic grants from Mr. Harold Simmons, Mr. Louis Chang, Dr. Joseph Lee and AVEO, Inc. (to K.K.-Z.). 


\section{Author Contributions}

Dr. Elani Streja had full access to all the data in the study and takes responsibility for the integrity of the data and the accuracy of the data analysis. R.E., Y.O., K.K.-Z., and E.S.: study concept and design. M.S., K.-Z., and E.S.: acquisition, analysis, or interpretation of data. R.E. and Y.O.: drafting of the manuscript. M.S., C.M.R., C.P.K., K.K.-Z., and E.S.: critical revision of the manuscript content for important intellectual content. R.E., Y.O., M.S., and E.S.: statistical analysis. R.E., Y.O., M.S., C.M.R., C.P.K., K.K.-Z., and E.S.: approving final version of manuscript.

\section{References}

1 United States Renal Data System. 2018 USRDS annual data report: Epidemiology of kidney disease in the United States. National Institutes of Health, NationalInstitute ofDiabetes and Digestive and Kidney Diseases, Bethesda, MD, 2018.

2 Collins AJ, Foley RN, Gilbertson DT, Chen SC. United States Renal Data System public health surveillance of chronic kidney disease and end-stage renal disease. Kidney Int Suppl (2011). 2015 Jun;5(1):2-7.

3 Lowrie EG, Lew NL. Death risk in hemodialysis patients: the predictive value of commonly measured variables and an evaluation of death rate differences between facilities. Am J Kidney Dis. 1990 May;15(5):458-82.

4 Iseki K, Uehara H, Nishime K, Tokuyama K, Yoshihara K, Kinjo K, et al. Impact of the initial levels of laboratory variables on survival in chronic dialysis patients. Am J Kidney Dis. 1996 Oct;28(4):541-8.

5 Duke M. Thiazide-induced hypokalemia. Association with acute myocardial infarction and ventricular fibrillation. JAMA. 1978 Jan; 239(1):43-5.

6 Hulting J. In-hospital ventricular fibrillation and its relation to serum potassium. Acta Med Scand Suppl. 1981;647 S647:109-16.

7 Quick G, Bastani B. Prolonged asystolic hyperkalemic cardiac arrest with no neurologic sequelae. Ann Emerg Med. 1994 Aug;24(2): 305-11.

8 Lawton JM. Hyperkalemic electromechanical dissociation. Wis Med J. 1990 Aug;89(8):45961.

9 Schrier RW, Regal EM. Influence of aldosterone on sodium, water and potassium metabolism in chronic renal disease. Kidney Int. 1972 Mar;1(3):156-68.

10 Gonick HC, Kleeman CR, Rubini ME, Maxwell $\mathrm{MH}$. Functional impairment in chronic renal disease. 3. Studies of potassium excretion. Am J Med Sci. 1971 May;261(5):281-90.

11 Torlén K, Kalantar-Zadeh K, Molnar MZ, Vashistha T, Mehrotra R. Serum potassium and cause-specific mortality in a large peritoneal dialysis cohort. Clin J Am Soc Nephrol. 2012 Aug;7(8):1272-84.

12 Selby NM, McIntyre CW. Peritoneal dialysis is not associated with myocardial stunning. Perit Dial Int. 2011 Jan-Feb;31(1):27-33.

13 Hayes J, Kalantar-Zadeh K, Lu JL, Turban S, Anderson JE, Kovesdy CP. Association of hypo- and hyperkalemia with disease progression and mortality in males with chronic kidney disease: the role of race. Nephron Clin Pract. 2012;120(1):c8-16.

Potassium and Mortality across Race in $\mathrm{PD}$
14 Chen Y, Sang Y, Ballew SH, Tin A, Chang AR, Matsushita K, et al. Race, Serum Potassium, and Associations With ESRD and Mortality. Am J Kidney Dis. 2017 Aug;70(2):244-51.

15 Kim T, Rhee CM, Streja E, Soohoo M, Obi Y, Chou JA, et al. Racial and Ethnic Differences in Mortality Associated with Serum Potassium in a Large Hemodialysis Cohort. Am J Nephrol. 2017;45(6):509-21.

16 Kuttykrishnan S, Kalantar-Zadeh K, Arah OA, Cheung AK, Brunelli S, Heagerty PJ, et al. Predictors of treatment with dialysis modalities in observational studies for comparative effectiveness research. Nephrol Dial Transplant. 2015 Jul;30(7):1208-17.

17 Podrid PJ, Fuchs T, Candinas R. Role of the sympathetic nervous system in the genesis of ventricular arrhythmia. Circulation. 1990 Aug;82(2 Suppl):I103-13.

18 Osadchii OE. Mechanisms of hypokalemiainduced ventricular arrhythmogenicity. Fundam Clin Pharmacol. 2010 Oct;24(5):547-59.

19 SmithNL,LemaitreRN,HeckbertSR,KaplanRC, TirschwellDL,LongstrethWT,etal.Serumpotassiumandstrokeriskamongtreatedhypertensive adults.AmJHypertens.2003Oct;16(10):806-13.

20 Lindinger MI. Potassium regulation during exercise and recovery in humans: implications for skeletal and cardiac muscle. J Mol Cell Cardiol. 1995 Apr;27(4):1011-22.

21 Gallen IW, Rosa RM, Esparaz DY, Young JB, Robertson GL, Batlle D, et al. On the mechanism of the effects of potassium restriction on blood pressure and renal sodium retention. Am J Kidney Dis. 1998 Jan;31(1):19-27.

22 Morris RC Jr, Sebastian A, Forman A, Tanaka $\mathrm{M}$, Schmidlin $\mathrm{O}$. Normotensive salt sensitivity: effects of race and dietary potassium. Hypertension. 1999 Jan;33(1):18-23.

23 Voors AW, Dalferes ER Jr, Frank GC, Aristimuno GG, Berenson GS. Relation between ingested potassium and sodium balance in young Blacks and whites. Am J Clin Nutr. 1983 Apr;37(4):583-94.

24 Aviv A, Hollenberg NK, Weder A. Urinary potassium excretion and sodium sensitivity in blacks. Hypertension. 2004 Apr;43(4):707-13.

25 Suh A, DeJesus E, Rosner K, Lerma E, Yu W, Young JB, et al. Racial differences in potassium disposal. Kidney Int. 2004 Sep;66(3):1076-81.

26 Taylor EN, Curhan GC. Differences in 24hour urine composition between black and white women. J Am Soc Nephrol. 2007 Feb; 18(2):654-9.

27 Turban S, Miller ER 3rd, Ange B, Appel LJ. Racial differences in urinary potassium excretion. J Am Soc Nephrol. 2008 Jul;19(7):1396-402.
28 Noori N, Kalantar-Zadeh K, Kovesdy CP, Murali SB, Bross R, Nissenson AR, et al. Dietary potassium intake and mortality in longterm hemodialysis patients. Am J Kidney Dis. 2010 Aug;56(2):338-47.

29 St-Jules DE, Goldfarb DS, Sevick MA. Nutrient Non-equivalence: Does Restricting HighPotassium Plant Foods Help to Prevent Hyperkalemia in Hemodialysis Patients? J Ren Nutr. 2016 Sep;26(5):282-7.

30 Barlow RJ, Connell MA, Milne FJ. A study of 48-hour faecal and urinary electrolyte excretion in normotensive black and white South African males. J Hypertens. 1986 Apr;4(2): 197-200.

31 Hedayati SS, Minhajuddin AT, Ijaz A, Moe OW, Elsayed EF, Reilly RF, et al. Association of urinary sodium/potassium ratio with blood pressure: sex and racial differences. Clin J Am Soc Nephrol. 2012 Feb;7(2):315-22.

32 Pratt JH, Jones JJ, Miller JZ, Wagner MA, Fineberg NS. Racial differences in aldosterone excretion and plasma aldosterone concentrations in children. N Engl J Med. 1989 Oct; 321(17):1152-7.

33 Szerlip HM, Weiss J, Singer I. Profound hyperkalemia without electrocardiographic manifestations. Am J Kidney Dis. 1986 Jun;7(6):461-5.

34 Aslam S, Friedman EA, Ifudu O. Electrocardiography is unreliable in detecting potentially lethal hyperkalaemia in haemodialysis patients. Nephrol Dial Transplant. 2002 Sep; 17(9):1639-42.

35 Einhorn LM, Zhan M, Hsu VD, Walker LD, Moen MF, Seliger SL, et al. The frequency of hyperkalemia and its significance in chronic kidney disease. Arch Intern Med. 2009 Jun; 169(12):1156-62.

36 Ackerman MJ, Tester DJ, Jones GS, Will ML, Burrow CR, Curran ME. Ethnic differences in cardiac potassium channel variants: implications for genetic susceptibility to sudden cardiac death and genetic testing for congenital long QT syndrome. Mayo Clin Proc. 2003 Dec;78(12):1479-87.

37 Goraya N, Munoz-Maldonado Y, Simoni J, Wesson DE. Fruit and Vegetable Treatment of Chronic Kidney Disease-Related Metabolic Acidosis Reduces Cardiovascular Risk Better than Sodium Bicarbonate. Am J Nephrol. 2019;49(6):438-48.

38 Saglimbene VM, Wong G, Ruospo M, Palmer SC, Garcia-Larsen V, Natale P, et al. Fruit and Vegetable Intake and Mortality in Adults undergoing Maintenance Hemodialysis. Clin J Am Soc Nephrol. 2019;14(2):250-60. 